\title{
Improving oral healthcare: towards measurement?
}

\author{
F. Baâdoudi, ${ }^{* 1}$ N. Maskrey, ${ }^{1,2}$ S. Listl, ${ }^{3,4}$ G. J. M. G. van der Heijden ${ }^{1}$ and D. Duijster ${ }^{1}$
}

In brief

Provides an overview of where dentistry is in a time of changing expectations and needs.
Highlights that consistent and comparable data on treatments, prevention and oral health outcomes are not routinely collected, analysed and made available.
Proposes that measures, by providing greater insight into care processes and outcomes, have the potential to improve oral healthcare.

In the last 30 years oral healthcare is increasingly being contested. Needs and expectations have evolved to a point of advanced restorative techniques, long term planning for preventative care and evidence-based practice, and patients and payers increasingly require more explicit demonstration of competence. However, relevant data are currently not routinely collected that would support approaches needed to further improve oral health and care. This paper sets out the case to collect and evaluate routinely available data. Variations in care and oral health could be described, which in turn would provide valuable insights for all wishing to further improve oral health and care.

\section{Explicit or implicit competence?}

Patients increasingly ask: 'Is this the best treatment for me?' and 'Should I get a second opinion?'

Implicit assumption of competence of healthcare professionals is no longer taken for granted. Healthcare is now characterised by accountability, scrutiny, measurement, incentives and markets. ${ }^{1}$ As a result of increased availability of information on the internet and multiple examples of variation and poor quality in healthcare, there is an international societal trend requiring explicit demonstration of competence. ${ }^{2,3}$

\section{Where is dentistry in this?}

Dentistry cannot be immune from this trend. In the previous century, the predominant professional paradigm in dentistry rested on

${ }^{1}$ Department of Social Dentistry, Academic Centre for Dentistry Amsterdam, University of Amsterdam and VU University, Gustav Mahlerlaan 3,004, 1081LA Amsterdam, The Netherlands; ${ }^{2}$ School of Pharmacy, Keele University, Newcastle under Lyme, Staffordshire ST5 5BG, United Kingdom; ${ }^{3}$ Department of Conservative Dentistry, Translational Health Economics Group, Heidelberg University,

Im Neuenheimer Feld 400, 69,120 Heidelberg, Germany; ${ }^{4}$ Department of Quality and Safety of Oral Health Care, Radboud University Nijmegen, 6525GD Nijmegen, The Netherlands.

${ }^{*}$ Correspondence to: F. Baâdoudi

Email: f.baadoudi@acta.nl

Refereed Paper. Accepted 15 September 2016 DOI: 10.1038/sj.bdj.2016.813

${ }^{\oplus}$ British Dental Journal 2016; 221: 547-548 special skills and technical knowledge owned by the dentist, and society granted the professionals the privilege to judge and self-regulate the quality of their own work. However, in the last 30 years, oral healthcare has become more contested and new challenges have arisen. Development of advanced restorative materials and techniques, together with a wider awareness of the benefits of preventative care, have increased the complexity of decision making for and with individual patients. Many health systems focused on providing curative, episodic care with the emphasis on treating current symptoms, while during the last decade the profession has been moving towards long term care planning and preventative care. ${ }^{4}$ Furthermore, patients' expectations have changed as many hold aesthetics as an equal alongside functionality and freedom from pain. ${ }^{5,6}$ The wider approach to consumerism in society is reflected in oral healthcare.

At the same time, evidence-based oral health practice has been encouraged. Dentists are expected to base their decisions on the best available information coupled with clinical expertise and the values and preferences of patients. ${ }^{7}$ This might seem straightforward in theory, but in practice it has proven difficult. Greenhalgh et al. speak of 'a movement in crisis.' The enormous volume of literature is impossible for a clinician to read, digest and apply to patient care, and there are serious concerns that the evidence is biased due to research often being funded by commercial companies using surrogate outcomes. ${ }^{9}$ In a policy review on oral healthcare the Dutch Health Council recently commented that the predominant focus of research in dentistry is on basic biomedical sciences and the development of new materials, ${ }^{10}$ rather than on high quality trials with patient-orientated outcomes, such as relief of oral disease manifestations and symptoms, and improved oral health and quality of life during long term follow-up. ${ }^{11,12}$ Furthermore, evidence-based practice is sometimes erroneously portrayed as a 'best approach' for everyone. However, evidence can only describe what is the best treatment, on average, for a population, and not for an individual.

With these changed expectations, how does dentistry meet the increased demand for explicit, transparent demonstration of safe, effective and evidence-based oral healthcare?

\section{Where is the data?}

Dentists, patients and policy makers may not be aware of variation of treatment, prevention and care provided by different dentists, in different localities and the various healthcare systems in different countries. This is because consistent and comparable data on treatments, prevention and oral health outcomes are simply not routinely collected, analysed and made available.

As a result, dentists lack data on the consistency of their care with the available best evidence, and how they are performing in 
comparison with other practitioners. Therefore it is difficult to answer questions such as: 'How does the care they provide compare to that provided by their fellow dentists?' and 'Does the care they provide satisfy the needs of their patients?' Comparative data of oral health and oral healthcare may illustrate where further development of oral healthcare is needed and its alignment with best evidence. While addressing the current absence of such data, the development of a set of relevant, valid and important measures is required as descriptors of practice performance and oral health outcomes.

\section{How do we make progress?}

A robust and comprehensive methodology should be used to construct measures, to ensure routinely available and comparable data. Dentists, patients, insurers and policy makers all have their role in improving healthcare and should therefore be included in the development of measures. Earlier attempts to provide more insight and transparency should be taken into account. For example, in the UK, where there is a pay for performance scheme for general practice, emphasis has been put on monitoring the processes of healthcare; this has led to distortion of clinical priorities with a focus too heavy on the means and not on the results of care. ${ }^{13,14} \mathrm{~A}$ balance is required in measurement - the data should describe care in ways that will benefit both professional and patient. In medicine, Berwick recommends a reduction in intensive mandatory measurements, ceasing complex individual (financial) incentives, shifting the strategy to quality rather than revenue, encouraging team work and skill mix towards a collective approach, using improvement science to spread better practice, protecting civility when discussing practice data, hearing the voice of patients routinely and rejecting greed. ${ }^{1}$

So, as dentistry sets out on this road it is important to accept that data and measures rarely provide definitive answers as to 'good' and 'bad' practice, and these answers should not be looked for; every practice and every individual decision with a patient is unique. What is considered highly relevant care for one patient, might in an identical clinical situation with the next patient be considered inappropriate. Moreover, when using data and measures to compare practice, it is important to consider socio-economic and demographic determinants of health. ${ }^{15,16}$ Data and measures should be used judiciously, avoiding normative approaches. In particular, they should be used to inform conversations on the one hand between oral health professionals on provided care and on the other hand between oral health professionals and patients. The aim should be to enable oral healthcare professionals to learn from their own practice data and move further towards a patient-centred and prevention-oriented service.

\section{Conclusion}

Improving oral healthcare delivery in the twenty-first century will require in many countries a more transparent, evidence-based and patient-centred care system. This can be facilitated by the construction of oral health measures based on a comprehensive and robust methodology, which should be applied to routinely collect data describing delivery and outcomes of care. All stakeholders, notably patients, professionals and policymakers, should be involved in the construction and deployment of comparative measures. To facilitate conversations on transparency of delivery and outcomes of oral healthcare between practices, patients and policy makers, an open and safe culture is required. Data and measures are to inform and for education, not judgement.

'Soft' as opposed to 'hard' approaches to improve practice ${ }^{17}$ should dominate - with practitioners themselves identifying good practice and areas for improvement. This should avoid the pitfalls of the paternalistic approach on accountability, scrutiny and measurement, which may easily result in feelings of suspicion, resistance and helplessness in healthcare professionals - the very people that the data should be helping. ${ }^{1}$

\section{Conflicts of interest}

All authors have no personal, commercial, political, or interests to declare. All authors receive funding from a European Union research grant (ADVOCATE) under Horizon 2020; grant agreement No. 635,183.

1. Berwick D. Era 3 for medicine and health care. JAMA 2016; 315: 1-2.

2. James $\mathrm{S}$. Understanding social factors and inequalities in health : 20th Century progress and 21st Century prospects. J Health Soc Behav 2002: 43: 125-142.

3. Baker A. Book: Crossing the quality chasm: A new health system for the 21st Century. BMJ 2001; 323: 1192-1192.

4. Hayashi M, Haapasalo M, Imazato S et al. Dentistry in the 21st century: challenges of a globalising world. Int Dent J 2014; 64: 333-342.

5. Hussain A, Khan F. History of dentistry. Arch Med Heal Sci 2014; 2: 106.

6. Owen H. Simulation in healthcare education. Switzerland: Springer, 2016.

7. Sackett D L, Rosenberg W M C, Grey J A M, Haynes R B, Richardson W S. Evidence based medicine: what it is and what it isn't. BMJ 1996; 312: 71-72.

8. Greenhalgh T, Howick J, Maskrey N. Evidence based medicine: a movement in crisis? BMJ 2014; 348: g3725.

9. Kearns C E, Glantz S A, Schmidt L A. Sugar industry influence on the scientific agenda of the National Institute of Dental Research's 1971 National Caries Program: a historical analysis of internal documents. PLoS Med 2015: 12: e1001798.

10. Gezondheidsraad. De mondzorg van morgen. Den Haag; 2012.

11. Kay E J, Nuttall N M, Kniil-Jones R. Restorative treatment thresholds and agreement in treatment decision-making Community Dent Oral Epidemiol 1992; 20: 265-268.

12. Mejàre I A, Klingberg G, Mowafi F K, Stecksén-Blicks C, Twetman S H A, Tranæus SH. A systematic map of systematic reviews in paediatric dentistry-what do we really know? PLoS One 2015; 10: e0117537.

13. Harris R, Bridgman C, Ahmad M et al. Introducing care pathway commissioning to primary dental care: measuring performance. Br Dent J 2011; 211: E22-E22.

14. Department of Health. Liberating the NHS: transparency in outcomes- a framework for the NHS: a consultation on proposals. London; 2010.

15. Gupta E, Robinson P G, Marya C M, Baker S R. Oral health inequalities: Relationships between environmental and individual factors. J Dent Res. 2015; DOI:10.1177/0022034515592880

16. Costa S M, Martins C C, Bonfim M de LC et al. A systematic review of socioeconomic indicators and dental caries in adults. Int J Environ Res Public Health; 2012; 9: 3540-3574.

17. Greenhalgh T, Robert G, Macfarlane F, Bate P, Kyriakidou 0 . Diffusion of innovations in service organizations: systematic review and recommendations. Milbank $Q$ 2004; 82: 581-629. 